\title{
Iterative Algebraic Soft-Decision List Decoding of Reed-Solomon Codes
}

\author{
Mostafa El-Khamy, Student, IEEE, and Robert J. McEliece, Fellow, IEEE
}

\begin{abstract}
In this paper, we present an iterative soft-decision decoding algorithm for Reed-Solomon (RS) codes offering both complexity and performance advantages over previously known decoding algorithms. Our algorithm is a list decoding algorithm which combines two powerful soft-decision decoding techniques which were previously regarded in the literature as competitive, namely, the Koetter-Vardy algebraic soft-decision decoding algorithm and belief-propagation based on adaptive parity-check matrices, recently proposed by Jiang and Narayanan. Building on the Jiang-Narayanan algorithm, we present a belief-propagation-based algorithm with a significant reduction in computational complexity. We introduce the concept of using a belief-propagation-based decoder to enhance the soft-input information prior to decoding with an algebraic soft-decision decoder. Our algorithm can also be viewed as an interpolation multiplicity assignment scheme for algebraic soft-decision decoding of RS codes.
\end{abstract}

Index Terms-Belief propagation, interpolation multiplicity, list decoding iterative decoding, maximum-likelihood (ML) decoding, Reed-Solomon (RS) codes, soft-decision decoding.

\section{INTRODUCTION}

$\mathbf{R}$ EED-SOLOMON (RS) codes [1] are among the most celebrated forward error correcting codes. The RS codes are currently used in a wide variety of applications, ranging from satellite communications to data storage systems. RS codes have been adopted as outer codes in the third-generation (3G) wireless standard, CDMA2000 high-rate broadcast packet data air interface [2], and are expected to be used as outer codes in concatenated coding schemes for future fourth-generation $(4 \mathrm{G})$ wireless systems.

Maximum-likelihood (ML) decoding of linear codes, in general, and RS codes, in particular, is NP-hard [3], [4]. It remains an open problem to find polynomial-time decoding algorithms with near ML performance. A soft-decision ML decoding algorithm was proposed by Vardy and Be'ery [5]. Further modifications of this algorithm were also studied [6]. Guruswami and Sudan (GS) [7], [8] invented a polynomial-time list decoding algorithm for RS codes capable of correcting beyond half the minimum distance of the code. Koetter and Vardy (KV) [9] developed an algebraic soft-decision decoding (ASD) algorithm for RS codes based on a multiplicity assignment scheme for the GS

Manuscript received January 16, 2005; revised April 22, 2005. This work was supported in part by the National Science Foundation (NSF) under Grant CCR-0118670 and in part by grants from Sony, Qualcomm, and the Lee Center for Advanced Networking. This paper was presented in part at the International Symposium on Information Theory and its Applications, Parma, Italy, October 2004.

The authors are with the Department of Electrical Engineering, California Institute of Technology, Pasadena, CA 91125 USA (e-mail: mostafa@systems.caltech.edu; rjm@systems. caltech.edu).

Digital Object Identifier 10.1109/JSAC.2005.862399 algorithm. Alternative ASD algorithms, such as the Gaussian approximation algorithm by Parvaresh and Vardy [10] and the algorithm by El-Khamy and McEliece based on the Chernoff bound [11], [12], have better performance.

Jiang and Narayanan (JN) developed an iterative algorithm based on belief propagation for soft decoding of RS codes [13], [14]. This algorithm compares favorably with other soft-decision decoding algorithms for RS codes and is a major step toward message passing decoding algorithms for RS codes. In the $\mathrm{JN}$ algorithm, belief propagation is run on an adapted parity-check matrix, where the columns in the parity-check matrix corresponding to the least reliable independent bits are reduced to an identity submatrix [13], [14]. The order statistics decoding algorithm by Fossorier and Lin [15] also sorts the received bits with respect to their reliabilities and reduces the columns in the generator matrix corresponding to the most reliable bits to an identity submatrix. This matrix is then used to generate (permuted) codewords using the most reliable bits. Other soft-decoding algorithms for RS codes include the generalized minimum distance (GMD) decoding algorithm introduced by Forney [16], the Chase II algorithm [17], the combined Chase II-GMD algorithm [18], and successive erasure-error decoding [19].

In this paper, we develop an algebraic soft-decision list decoding algorithm based on the idea that belief propagation-based algorithms could be deployed to improve the reliability of the symbols that is then utilized by an interpolation multiplicity assignment algorithm. Our algorithm combines the KV and the JN algorithms. An outline of this paper is as follows. Some preliminaries are given in Section II. In Section III, we briefly review algebraic soft-decoding algorithms, in general, and the KV algorithm, in particular. The JN algorithm is explained in the context of this paper in Section IV. Some modifications to the JN algorithm are introduced in Section V. One of the main contributions in this paper, the iterative algebraic soft-decision list decoding algorithm, is presented in Section VI. Another main contribution, a low complexity algorithm based on the JN algorithm, is presented in Section VII. Some discussions, as well as some numerical results are presented in Section VIII. Finally, we conclude this paper in Section IX and suggest future research directions.

\section{PRELIMINARIES}

Throughout this paper, $\boldsymbol{d}=\left[d_{0}, d_{1}, \ldots, d_{k-1}\right]$ will denote a $k$-dimensional vector over $F_{q}$, where $F_{q}$ is the finite field of $q$ elements. $\mathcal{C}$ will denote an $(n, k) \mathrm{RS}$ code. An $(n, k) \mathrm{RS}$ codeword $\boldsymbol{u}=\left[u_{0}, u_{1}, \ldots, u_{n-1}\right]$ could be generated by evaluating the data polynomial $D(x)=\sum_{i=0}^{k-1} d_{i} x^{i}$ at $n$ elements of the 
field composing a set, called the support set of the code. This set is vital for the operation of the Guruswami-Sudan algorithm. Let $\alpha$ be a primitive element in $F_{q}$. Since the polynomial $U(x)=\sum_{i=0}^{n-1} u_{i} x^{i}$ associated with the codeword $\boldsymbol{u} \in \mathcal{C}$ generated by polynomial evaluation has $\alpha, \alpha^{2}, \ldots, \alpha^{n-k}$ as zeros [20], a valid parity-check matrix for $\mathcal{C}$ is [21]

$$
\mathcal{H}=\left[\begin{array}{cccc}
1 & \alpha & \ldots & \alpha^{n-1} \\
1 & \alpha^{2} & \ldots & \alpha^{2(n-1)} \\
\vdots & \vdots & \ldots & \vdots \\
1 & \alpha^{n-k} & \ldots & \alpha^{(n-k)(n-1)}
\end{array}\right]
$$

The redundancy of the code's binary image will be denoted by $\tilde{r}$, where $\tilde{r}=\tilde{n}-\tilde{k}$ and $\tilde{n}=m n$ and $\tilde{k}=m k$. The results in this paper assume that the binary image and the corresponding binary parity-check matrix are of the form described here. Let $p(x)$ be a primitive polynomial in $F_{2}[x]$ and $C$ be its companion matrix [22]. The companion matrix is an $m \times m$ binary matrix. Since the mapping $\alpha^{i} \leftrightarrow C^{i},\{i=0,1,2, \ldots\}$ induces a field isomorphism, an $\tilde{r} \times \tilde{n}$ binary parity-check matrix $H$ is obtained by replacing every element $\alpha^{i}$ in the parity-check matrix $\mathcal{H}$ by its corresponding $m \times m$ matrix $\mathcal{C}^{i}$. The binary image $\boldsymbol{b}$, such that $H \boldsymbol{b}^{T}=0$, is obtained by representing each element $u_{j} \in F_{2^{m}}$ with $u_{j}=u_{j, 0}+u_{j, 1} \alpha+\cdots+u_{j, m-1} \alpha^{m-1}$, where $u_{j, i} \in F_{2}$.

An $q \times n$ array of real numbers will be denoted by $W=$ $\left[W_{i}(\beta)\right]$, where $i=0,1, \ldots, n-1$ and $\beta \in F_{q}$. If $\boldsymbol{u}$ is transmitted and the corresponding channel output is $\boldsymbol{y}$, then we denote the a posteriori probabilities $\operatorname{Pr}\left\{u_{i}=\beta \mid y_{i}\right\}$ by $\Pi_{i}(\beta)$.

\section{Algebraic Soft DECODING}

An algebraic soft decoder makes use of the soft information available from the channel. Given the a posteriori probability matrix $\Pi$, a multiplicity assignment algorithm generates an $q \times n$ multiplicity matrix $M=\left[M_{i}(\beta)\right]$ of nonnegative integers. The interpolation cost of $M$ is defined to be ${ }^{1}|M| \triangleq$ $1 / 2 \sum_{i=0}^{n-1} \sum_{\beta \in F_{q}} W_{i}(\beta)\left(W_{i}(\beta)+1\right)$ and the score of $\boldsymbol{u}$ with respect to $M$ is $\langle\boldsymbol{u}, M\rangle \triangleq \sum_{i=0}^{n-1} M_{i}\left(u_{i}\right)$. This multiplicity matrix is then passed to a (modified) GS algorithm consisting of two main steps [7], [23].

Step 1) Interpolation: Construct a bivariate polynomial $Q(x, y)$ of minimum $(1, k-1)$ weighted degree that passes through each of the points $\left(T_{i}, \beta\right)$ with multiplicity $M_{i}(\beta)$, where $\beta \in F_{q}$ and $i=0,1, \ldots, n-1$.

Step 2) Factorization: Find all linear factors $(y-$ $G(x)) \mid Q(x, y)$, where $G(x)$ is a polynomial of degree less than $k$. Each such polynomial $G(x)$ is placed on the list.

A solution to the interpolation problem exists if $|M|$ is strictly less than the number of monomials in $Q$ such that $Q$ is of minimal $(1, k-1)$ weighted degree $\Delta_{k-1}(|M|)$ [24]. A sufficient condition for a codeword $\boldsymbol{u}$ to be on the GS generated list is [7], [9]

$$
\langle\boldsymbol{u}, M\rangle>\Delta_{k-1}(|M|)
$$

${ }^{1}$ To prevent notational ambiguity, $\|x\|_{1}$ will denote the magnitude of $x$. where $\Delta_{v}(\gamma)=\lfloor\gamma / m+v(m-1) / 2\rfloor$ for $m=$
$\lfloor\sqrt{2 \gamma / v+1 / 4}+1 / 2\rfloor[11]$. In case the cost tends to infinity, the sufficient condition is [9], [11]

$$
\frac{\langle\boldsymbol{u}, M\rangle}{\|M\|_{2}}>\sqrt{k-1} .
$$

In this paragraph, we briefly review well-known ASD algorithms. For more details, we refer the readers to the given references. The KV algorithm maximizes the mean of the score. A reduced complexity $\mathrm{KV}$ algorithm constructs the multiplicity matrix $M$ as follows [9], [25]:

$$
M_{i}(\beta)=\left\lfloor\lambda \Pi_{i}(\beta)\right\rfloor
$$

where $\lambda>0$ is a complexity parameter determined by $|M|$. For $|M|=\gamma$, it can be shown that $\lambda=(-1+\sqrt{1+8 \gamma / n}) / 2$. Other algorithms of [10] and [11] minimize the error probability directly. The algorithm of [10] (Gauss) assumes a Gaussian distribution of the score, while that of [11] (Chernoff) minimizes a Chernoff bound on the error probability. The later appears to have the best performance.

\section{AdAPtive Belief Propagation}

Gallager devised an iterative algorithm for decoding his lowdensity parity-check (LDPC) codes [26]. This algorithm was the first appearance in the literature of what we now call belief propagation (BP). Recall that $H$ is the parity-check matrix associated with the binary image of the RS code. It has $\tilde{r}$ rows corresponding to the check nodes and $\tilde{n}$ columns corresponding to the variable nodes (transmitted bits). $H_{i, j}$ will denote the element in the $i$ th row and $j$ th column of $H$. Define the sets, $J(i) \triangleq\left\{j \mid H_{i, j}=1\right\}$ and $I(j) \triangleq\left\{i \mid H_{i, j}=1\right\}$. Define $Q_{i, j}$ to be the log-likelihood ratio (LLR) of the $j$ th symbol, $u_{j}$, given the information about all parity-check nodes except node $i$ and $R_{i, j}$ to be the LLR that check node $i$ is satisfied when $u_{j}$ is fixed to 0 and 1 , respectively. Given the vector $\boldsymbol{\Lambda}^{\text {in }}$ of initial LLRs, the BP algorithm outputs the extrinsic LLR's $\boldsymbol{\Lambda}^{\boldsymbol{x}}$ as described below [27], [28].

Algorithm 1: Damped Log Belief Propagation (LBP)

For all $(i, j)$ such that $H_{i, j}=1$ :

Initialization: $Q_{i, j}=\Lambda_{j}^{\text {in }}$

DO

Horizontal Step:

$$
\begin{aligned}
R_{i, j} & =\log \left(\frac{1+\prod_{k \in J(i) \backslash j} \tanh \left(\frac{Q_{i, k}}{2}\right)}{1-\prod_{k \in J(i) \backslash j} \tanh \left(\frac{Q_{i, k}}{2}\right)}\right) \\
& =2 \tanh ^{-1}\left(\prod_{k \in J(i) \backslash j} \tanh \left(\frac{Q_{i, k}}{2}\right)\right) .
\end{aligned}
$$

Vertical Step:

$$
Q_{i, j}=\Lambda_{j}^{\text {in }}+\theta \sum_{k \in I(j) \backslash i} R_{k, j} .
$$

While stopping criterion is not met.

Extrinsic Information: $\Lambda_{j}^{x}=\sum_{k \in I(j)} R_{k, j}$. 
The factor $\theta$ is termed the vertical step damping factor and $0<\theta \leq 1$. The magnitude of $\theta$ is determined by our level of confidence about the extrinsic information. In our implementations, $\theta$ is 0.5 . Equation (5) is specifically useful for fast hardware implementations, where the tanh function will be quantized to a reasonable accuracy and implemented as a lookup table. In our implementation, damped LBP is run for a small number of iterations on a fixed parity-check matrix, so the stopping criterion is the number of iterations. In case that only one LBP iteration is run on the parity-check matrix, the vertical step is eliminated.

Next, we describe the JN algorithm [13], [14], which builds on the BP algorithm. In the JN algorithm, BP is run on the parity-check matrix after reducing its independent columns corresponding to the least reliable bits to an identity submatrix. We will refer to such a class of algorithms, that adapt the paritycheck matrix before running BP, by adaptive belief propagation (ABP).

\section{Algorithm 2: The JN Algorithm}

\section{Initialization: $\Lambda^{p}:=\Lambda^{\text {ch }}$ DO}

1) Sort $\Lambda^{\boldsymbol{p}}$ in ascending order of magnitude and store the sorting index. The resulting vector of sorted LLRs is

$$
\boldsymbol{\Lambda}^{\text {in }}=\left[\Lambda_{1}^{\text {in }}, \Lambda_{2}^{\text {in }}, \ldots, \Lambda_{n m}^{\text {in }}\right]
$$

$\left\|\Lambda_{k}^{\mathrm{in}}\right\|_{1} \leq\left\|\Lambda_{k+1}^{\mathrm{in}}\right\|_{1}$ for $k=1,2, \ldots, n m-1$ and $\Lambda^{\text {in }}=$ $P \Lambda^{p}$, where $P$ defines a permutation matrix.

2) Rearrange the columns of the binary parity-check matrix $H$ to form a new matrix $H_{P}$, where the rearrangement is defined by the permutation $P$.

3) Perform Gaussian elimination (GE) on the matrix $H_{P}$ from left to right. GE will reduce the first independent $(n-k) m$ columns in $H_{P}$ to an identity submatrix. The columns which are dependent on previously reduced columns will remain intact. Let this new matrix be $\hat{H}_{P}$.

4) Run $\log$ BP on the parity-check matrix $\hat{H}_{P}$ with initial LLR's $\Lambda^{\text {in }}$ for a maximum number of iterations $I t_{H}$ and a vertical step damping factor $\theta$. The $\log$ BP algorithm outputs extrinsic LLR's $\boldsymbol{\Lambda}^{x}$.

5) Update the LLR's, $\boldsymbol{\Lambda}^{\boldsymbol{q}}=\boldsymbol{\Lambda}^{\mathrm{in}}+\alpha_{1} \boldsymbol{\Lambda}^{\boldsymbol{x}}$ and $\boldsymbol{\Lambda}^{\boldsymbol{p}}:=P^{-1} \boldsymbol{\Lambda}^{\boldsymbol{q}}$, where $0<\alpha_{1} \leq 1$ is called the ABP damping factor and $P^{-1}$ is the inverse of $P$.

6) Decode using $\Lambda^{p}$ as an input to the decoding algorithm $D$.

While Stopping criterion not satisfied.

The JN algorithm assumed that the decoder $D$ is one of the following hard-decision decoders:

- HD: Perform hard-decisions on the updated LLR's, $\hat{\boldsymbol{u}}=$ $\left(1-\operatorname{sign}\left(\boldsymbol{\Lambda}^{\boldsymbol{p}}\right)\right) / 2$. If $H \hat{\boldsymbol{u}}^{T}=0$, then a decoding success is signaled.

- BM: Run a bounded minimum distance decoder such as the Berlekamp-Massey (BM) algorithm on the LLRs after hard-decisions. If the BM algorithm finds a codeword, a decoding success is signaled.

The performance largely depends on the decoder $D$ and the stopping criterion used. This is discussed in Section V.

\section{Modifications to the JN Algorithm}

The stopping criterion deployed in the $\mathrm{JN}$ algorithm is as follows [14].

- Stop if a decoding success is signaled by the decoder $D$ or if the number of iterations is equal to the maximum number of iterations $N_{1}$.

We propose a list-decoding stopping criterion in which a list of codewords is iteratively generated. The list-decoding stopping criterion is as follows.

- If a decoding success is signaled by the decoder $D$, add the decoded codeword to a global list of codewords. Stop if the number of iterations is equal to the maximum number of iterations, $N_{1}$.

If more than one codeword is on the global list of codewords, then the list-decoder's output is the codeword which is at the minimum Euclidean distance from the received vector. Alternatively, one could only save the codeword with the largest conditional probability, given the received vector. This codeword would be the candidate for the list decoder's output when the iteration loop terminates.

The advantage of our proposed list-decoding stopping criterion over the stopping criterion in the $\mathrm{JN}$ algorithm is emphasized in the case of higher rate codes, where the decoder error probability is relatively high. Given a decoding algorithm $D$, the JN ABP algorithm may result in updating the received vector to lie in the decoding region of an erroneous codeword. However, running more iterations of the JN ABP algorithm may move the updated received vector into the decoding sphere of the transmitted codeword. The decoding algorithm $D$ should also be run on the channel LLRs before any ABP iteration is carried out. If the decoder succeeds to find a codeword, it is added to the list.

Jiang and Narayanan [13] proposed running $N_{2}$ parallel decoders (outer iterations), each with the $\mathrm{JN}$ stopping criterion and a maximum of $N_{1}$ inner iterations. Each one of these $N_{2}$ iterations (decoders) starts with a different random permutation of the sorted channel LLRs in the first inner iteration. The outputs of these $\mathrm{N}_{2}$ decoders form a list of at most $\mathrm{N}_{2}$ codewords. If each of these $\mathrm{N}_{2}$ decoders succeeds to find a codeword, the closest codeword to the received vector is chosen. We also run $N_{2}$ parallel decoders (outer iterations), each with the list-decoding stopping criterion, to form a global list of at most $N_{1} N_{2}$ codewords. We propose doing the initial sorting of the channel LLRs in a systematic way to ensure that most bits will have a chance of being in the identity submatrix of the adapted parity-check matrix. The improved performance achieved by these restarts could be explained by reasoning that if a higher reliability bit is in error, then it has a higher chance of being corrected if its corresponding column in the parity-check matrix is in the sparse identity submatrix.

Let $z=\left\lfloor\tilde{n} / N_{2}\right\rfloor$, then at the $(j+1)$ th outer iteration, $j>0$, the initial LLR vector at the first inner iteration is

$$
\left[\Lambda_{j z+1}^{\text {in }}, \ldots, \Lambda_{(j+1) z}^{\text {in }}, \Lambda_{1}^{\text {in }}, \ldots, \Lambda_{j z}^{\text {in }}, \Lambda_{(j+1) z+1}^{\text {in }}, \ldots, \Lambda_{\tilde{n}}^{\text {in }}\right]
$$

where $\boldsymbol{\Lambda}^{\text {in }}$ is the vector of sorted channel LLRs. The columns of $H_{P}$ will also be rearranged according to the same permuatation. If $(j+1) z \leq \tilde{r}$, then it is less likely that this initial permutation will introduce new columns into the identity submatrix other 
than those which existed in the first outer iteration. After the first outer iteration, it is thus recommended to continue with the $(j+1)$ th outer iteration such that $(j+1)>\tilde{r} / z$.

Another modification that could improve the performance of the JN algorithm is to run a small number of iterations of damped log belief propagation on the same parity-check matrix. Although belief propagation is not exact due to the cycles in the associated Tanner graph, running a very small number of iterations of belief propagation is very effective [29]. Observing that the complexity of belief propagation is much lower than that of Gaussian elimination, one gets a performance enhancement at a slightly increased complexity.

Throughout the remaining of this paper, we will refer to the modified JN algorithm with a list decoding stopping criterion, as well as with the other modifications introduced in this section, by ABP-BM if the decoding algorithm $D$ is BM. Similarly, if the decoding algorithm was HD, the algorithm is referred to by ABP-HD. One of the main contribution in this paper, the utilization of the a posteriori probabilities at the output of the ABP algorithm as the soft information input to an ASD algorithm, is presented in Section VI.

\section{HYBRID ABP-ASD LiSt DECODING AlgORITHM}

Koetter and Vardy [9] point out that it is hard to maximize the mean of the score with respect to the to the true channel a posteriori probabilities. Previous multiplicity assignment algorithms [9]-[11] assumed approximate a posteriori probabilities. The problem is simplified by assuming that the transmitted codeword is drawn uniformly from $F_{q}^{n}$. Also, the $n$ received symbols are assumed to be independent and, thus, be assumed to be uniformly distributed. In such a case, the a posteriori probabilities are approximated to be a scaling of the channel transition probabilities

$$
\Pi_{i}^{\mathrm{ch}}(\beta)=\frac{\operatorname{Pr}\left\{y_{i} \mid u_{i}=\beta\right\}}{\sum_{\omega \in F_{q}} \operatorname{Pr}\left\{y_{i} \mid u_{i}=\omega\right\}} .
$$

However, from the maximum distance separable (MDS) property of RS codes, any $k$ symbols (only) are $k$-wise independent and could be treated as information symbols and, thus, uniformly distributed. Thus, these assumptions are more valid for higher rate codes and for memoryless channels. It is well known that belief propagation algorithms improve the reliability of the symbols by taking into account the geometry of the code and the correlation between symbols (see, for example, [27].) Due to the dense nature of the parity-check matrix of the binary image of RS codes, running belief propagation directly will not result in a good performance. Because the Tanner graph associated with the parity-check matrix of the binary image of RS codes has cycles, the marginals passed by the (log) belief propagation algorithm are no longer independent and the information starts to propagate in the loops.

Jiang and Narayanan [14] proposed a solution to this problem by adapting the parity-check matrix after each iteration. When updating the check node reliabilities $R_{i, j}$ [see (5)] corresponding to a pivot in a single weight column, the information $Q_{i, j}$ from any of the least reliable independent bits does not enter into the summation. One reason for the success of ABP is that the reliabilities of the least reliable bits are updated by only passing the information from the more reliable bits to them. An analytical model for belief propagation on adaptive parity-check matrices was recently proposed [30].

Our ABP-ASD algorithm is summarized by the following chain:

$$
\boldsymbol{u} \rightarrow \Pi^{\mathrm{ch}} \stackrel{\mathrm{ABP}}{\longrightarrow} \hat{\Pi} \underbrace{\stackrel{\mathcal{A}}{\longrightarrow} M \rightarrow}_{\mathrm{ASD}} \hat{\boldsymbol{u}}
$$

where $\boldsymbol{u}$ is the transmitted codeword, $\mathcal{A}$ is a multiplicity assignment algorithm, $M$ is the multiplicity matrix and $\hat{\boldsymbol{u}}$ is the decoder output. In particular, the ABP-ASD list decoder is implemented by deploying the list decoder stopping criterion, proposed in the previous section, with an ASD decoding algorithm $D$ (see Alg. 2).

- ASD: Using $\boldsymbol{\Lambda}^{\boldsymbol{p}}$, generate an $q \times n$ reliability matrix $\hat{\Pi}$ which is then used as an input to an multiplicity assignment algorithm to generate multiplicities according to the required interpolation cost. This multiplicity matrix is passed to the (modified) GS list decoding algorithm. If the generated codeword list is not empty, the list of codewords is augmented to the global list of codewords. If only one codeword is required, the codeword with the highest reliability with respect to the channel LLR's $\boldsymbol{\Lambda}^{\text {ch }}$ is added to the global list.

In this paper, the $\mathrm{KV}$ algorithm is used as the multiplicity assignment scheme. More efficient but more complex multiplicity assignment schemes could also be used [11]. The joint ABP-ASD algorithm corrects decoder failures (the received word does not lie in the decoding region centered around any codeword) of the ASD decoder $D$, by iteratively enhancing the reliabilities of the received word and, thus moving the received word into the decoding region around a certain codeword. The decoding region in turn depends on the algorithm $D$ and the designed interpolation cost. Furthermore, it attempts to eliminate decoder errors (the decoded codeword is not the transmitted codeword) by iteratively adding codewords to the global list of codewords and choosing the most probable one.

Since ASD is inherently a list decoding algorithm with a larger decoding region, it is expected that ABP-ASD outperforms ABP-HD and ABP-BM. Since our algorithm transforms the channel LLRs into interpolation multiplicities for the GS algorithm, then, by definition, it is an interpolation multiplicity assignment algorithm for ASD.

The ABP-ASD algorithm has a polynomial-time complexity. The ABP step involves $o\left(\tilde{n}^{2}\right)$ floating point operations, for sorting and $\mathrm{BP}$, and $o\left(\min \left(\tilde{k}^{2}, \tilde{r}^{2}\right) \tilde{n}\right)$ binary operations for GE [13]. As for ASD, the KV MA algorithm [see (4)] has a time complexity of $O\left(n^{2}\right)$. An efficient algorithm for solving the interpolation problem is Koetter's algorithm [23] with a time complexity of $O\left(n^{2} \lambda^{4}\right)$. A reduced complexity interpolation algorithm is given in [24]. Roth and Ruckenstein [31] proposed an efficient factorization algorithm with a time complexity $O\left(\left(l \log ^{2} l\right) k(n+l \log q)\right)$, where $l$ is an upper bound on the ASD's list size and is determined by $\lambda$. 


\section{LOW COMPLEXITY ABP ALGORITHM}

Most of the complexity of adaptive belief propagation lies in row reducing the binary parity-check matrix (after rearranging the columns according to the permutation $P$ ). To reduce the complexity one could make use of the columns already reduced in the previous iteration.

We will use the same notation as in Alg. 2 with a subscript $j$ to denote the values at iteration $j$. For example, the vector of sorted LLRs at the $j$ th iteration is $\boldsymbol{\Lambda}_{\boldsymbol{j}}^{\text {in }}$. Define $P_{j}(H)$ to be the matrix obtained when the columns of the parity-check matrix $H$ are permuted according to the permutation $P_{j}$ at the $j$ th iteration. $\mathrm{GE}(H)$ will be the reduced matrix (with an identity submatrix) after Gaussian elimination is carried out on the matrix $H$.

Let $R_{j} \triangleq\{t: t$ th column of $H$ was reduced to a column of unit weight in $\left.\operatorname{GE}\left(P_{j}(H)\right)\right\}$. It is clear that the cardinality of $R_{j}$ is $\tilde{r}$. Now, assume that $\log$ BP is run and that the LLRs are updated and inverse permuted to get $\boldsymbol{\Lambda}_{\boldsymbol{j}}^{\boldsymbol{p}}$ (step 5 in Alg. 2). The set of indices of the $\tilde{r}$ (independent) LLRs in $\boldsymbol{\Lambda}_{\boldsymbol{j}}^{\boldsymbol{p}}$ with the smallest magnitude will be denoted by $S_{j+1}$. By definition, $P_{j+1}$ is the permutation that sorts the LLRs in $\boldsymbol{\Lambda}_{\boldsymbol{j}}^{\boldsymbol{p}}$ in ascending order according to their magnitude to get $\boldsymbol{\Lambda}_{\boldsymbol{j}+\mathbf{1}}^{\text {in }}$. The set $U_{j+1} \triangleq R_{j} \bigcap S_{j+1}$ is, thus, the set of indices of bits which are among the least reliable independent bits at the $(j+1)$ th iteration and whose corresponding columns in the reduced parity-check matrix at the previous iteration were in the identity submatrix.

The algorithm is modified such that GE will be run on the matrix whose left most columns are those corresponding to $U_{j+1}$. To construct the identity submatrix, these columns may only require row permutations for arranging the pivots (ones) on the diagonal. Note that these permutations may have also been required when running GE on $P_{j+1}(H)$. Only a small fraction of the columns will need to be reduced to unit weight leading to a large reduction in the GE computational complexity. Also, note that what matters is that a column corresponding to a bit with low reliability lies in the identity (sparse) submatrix and not its position within the submatrix. This is justified by the fact that the update rules for all the LLRs corresponding to columns in the identity submatrix are the same. Thus, provided that the first $\tilde{r}$ columns in $P_{j+1}(H)$ are independent, changing their order does not alter the performance of the ABP algorithm. To summarize the proposed reduced complexity ABP algorithm can be stated as follows.

Algorithm 3: Low Complexity Adaptive Belief Propagation Initialization: $\Lambda^{p}:=\Lambda^{\text {ch }}, j=1$

\section{DO}

If $j=1$

Proceed as in the first iteration of Alg. $2 ; \Lambda_{\mathbf{1}}^{\text {in }}=\left.\Lambda^{\text {in }}\right|_{\text {Alg. } 2}, P_{1}=$ $\left.P\right|_{\mathrm{Alg} .2}, \hat{H}_{1}=\left.\hat{H}_{P}\right|_{\mathrm{Alg} .2}$ and $\boldsymbol{\Lambda}_{\mathbf{1}}^{\boldsymbol{q}}=\left.\boldsymbol{\Lambda}^{\boldsymbol{q}}\right|_{\mathrm{Alg} .2}$.

If $j>1$

1) Sort the updated LLR vector $\boldsymbol{\Lambda}_{\boldsymbol{j}-\mathbf{1}}^{\boldsymbol{q}}$ in ascending order of the magnitude of its elements. Let $W_{j}^{\prime}$ be the associated sorting permutation matrix.

2) Rearrange the columns of the binary parity-check matrix $\hat{H}_{j-1}$ to form a new matrix

$$
Q_{j}^{\prime}=W_{j}^{\prime}\left(\hat{H}_{j-1}\right)
$$

3) Rearrange the most left $\tilde{r}$ columns of the binary parity-check matrix $Q_{j}^{\prime}$ such that the columns of unit weight are the most left columns. Let $W_{j}^{\prime \prime}$ be the corresponding permutation matrix. (This could be done by sorting the first $\tilde{r}$ columns of $Q_{j}^{\prime}$ in ascending order according to their weight.) Let the resulting matrix be

$$
Q_{j}^{\prime \prime}=W_{j}^{\prime \prime}\left(Q_{j}^{\prime}\right)
$$

4) Permute the LLR vector;

$$
\Lambda_{j}^{\text {in }}=P_{j}^{\prime} \Lambda_{j-1}^{q}
$$

where $P_{j}^{\prime}=W_{j}^{\prime} W_{j}^{\prime \prime}$.

5) Update the (global) permutation matrix;

$$
P_{j}=P_{j}^{\prime} P_{j-1} .
$$

6) Run Gaussian elimination on the matrix $Q_{j}^{\prime \prime}$ from left to right;

$$
\hat{H}_{j}=\mathrm{GE}\left(Q_{j}^{\prime \prime}\right) .
$$

7) Run damped LBP on $\hat{H}_{j}$ with initial LLR's $\boldsymbol{\Lambda}_{j}^{\text {in }}$ for $I t_{H}$ iterations. The output vector of extrinsic LLRs is $\boldsymbol{\Lambda}_{\boldsymbol{j}}^{\boldsymbol{x}}$.

8) Update the LLRs;

$$
\boldsymbol{\Lambda}_{\boldsymbol{j}}^{\boldsymbol{q}}=\boldsymbol{\Lambda}_{\boldsymbol{j}}^{\mathrm{in}}+\alpha_{1} \boldsymbol{\Lambda}_{\boldsymbol{j}}^{\boldsymbol{x}} \text { and } \boldsymbol{\Lambda}_{\boldsymbol{j}}^{\boldsymbol{p}}=P_{j}^{-1} \boldsymbol{\Lambda}_{\boldsymbol{j}}^{\boldsymbol{q}}
$$

9) Decode using $\boldsymbol{\Lambda}_{\boldsymbol{j}}^{\boldsymbol{p}}$ as an the input to the decoding algorithm $D$.

10) Increment $j$.

While Stopping criterion not satisfied.

The algorithm as described above iteratively updates a global permutation matrix and avoids inverse permuting the row-reduced parity-check matrix in each iteration. The implementation of the algorithm also assumes for simplicity that the columns in the parity-check matrix corresponding to the $\tilde{r}$ least reliable bits are independent and could, therefore, be reduced to unit weight columns. It is also noticed that in practice the cardinality of $U_{j+1}$ is close to $\tilde{r}$ which means that the GE elimination complexity will be significant only in the first iteration.

We will assume the favorable condition in which the most left $\tilde{r}$ columns of an parity-check matrix are independent. Taking into account that the parity-check matrix is a binary matrix, the maximum number of binary operations required to reduce the first $\tilde{r}$ columns to an identity submatrix in the JN algorithm (Alg. 2) can be shown to be

$$
\Theta_{\mathrm{GE}}=2 \sum_{\alpha=1}^{\tilde{r}}(\tilde{r}-\alpha)(\tilde{n}-\alpha+1)<\tilde{r}^{2} \tilde{n}-\tilde{r} \tilde{k} .
$$

(It is assumed that the two GE steps, elimination and back substitution are symmetric). Row permutation operations were neglected. Now, assume that the cardinality of $U_{j+1}$ is $\delta \tilde{r}$, where $\delta \leq 1$. For the modified algorithm, only row permutations may be required for the first $\delta \tilde{r}$ columns to arrange the pivots on the 
diagonal of the identity submatrix. These permutations may also be required for the JN algorithm. Then, the relative reduction in complexity is

$$
\begin{aligned}
& \frac{\Theta_{\mathrm{GE}} \text { in Alg. } 2-\Theta_{\mathrm{GE}} \text { in Alg. } 3}{\Theta_{\mathrm{GE}} \text { in Alg. } 2} \\
& =\frac{\sum_{\alpha=1}^{\delta \tilde{r}}(\tilde{r}-\alpha)(\tilde{n}-\alpha+1)}{\sum_{\alpha=1}^{\tilde{r}}(\tilde{r}-\alpha)(\tilde{n}-\alpha+1)} \\
& \approx \frac{\left(\tilde{r}^{2} \tilde{n}\right)\left(2 \delta-\delta^{2}\right)-\delta \tilde{r} \tilde{k}}{\tilde{r}^{2} \tilde{n}-\tilde{r} \tilde{k}} \approx 2 \delta-\delta^{2} .
\end{aligned}
$$

For example, if we assume that on average $\delta=0.5$, a simple calculation for the $(255,239)$ code over $F_{256}$ shows that the relative reduction in the complexity of the GE step is about $75 \%$. In practice $\delta$ is close to one. Note that Alg. 3 does require sorting $\tilde{r}$ columns of $Q_{j}^{\prime}$ [see step (3)] according to their weight but the complexity is relatively small.

\section{NUMERICAL RESULTS AND DISCUSSIONS}

In Section VIII-A, a fast simulation setup is described for ABP list decoding. Bounds on the error probability of the ML decoder are then discussed. We then show simulation results for our algorithm.

\section{A. Fast Simulation Setup}

We describe a fast simulation setup for ABP with a list decoding stopping criterion. One could avoid running the actual decoder $D$ at each iteration and instead check whether the transmitted codeword is on the list generated by the decoder $D$. The stopping criterion would be modified such that the iterative decoding stops if the transmitted codeword is on the list or if the maximum number of iterations is reached. A decoding success is signaled if the transmitted codeword is on the list.

It is easy to see that this simulation setup is equivalent to running the actual $\mathrm{ABP}$ list decoder for the maximum number of iterations. Suppose that the received sequence results in an ML error, then it is very unlikely that the decoder $D$ will correctly decode the received word at any iteration. In case of an ML decoder success and the transmitted codeword is added to the global list at a certain iteration, which presumably could be checked, then it would be the closest codeword to the received word and, thus, the list decoder's choice. Thus, for a fast implementation, a decoding success is signaled and iteration stops once the transmitted codeword appears on the global list.

In case that $D$ is a bounded minimum distance decoder such as the Berlekamp-Massey (BM) algorithm, the transmitted codeword would be on the global list if it is at a Hamming distance of $\leq\lfloor n-k / 2\rfloor$ from the hard-decisioned (modified) LLRs. If $D$ is an ASD algorithm that assigns the multiplicity matrix $M$, the transmitted codeword is on the ASD's list (and, thus, the global list) if it satisfies the sufficient conditions of (2) and (3) for finite and infinite interpolation costs, respectively. It was shown in [9], that simulating the KV algorithm by checking the sufficient condition of (2) results in accurate results. This is partially justified by the fact that on average, the ASD's list size is one [32]. This is also justified by observing that if the ASD's list is empty (a decoding failure), the condition (2) will not be satisfied. However, if the list is nonempty but the transmitted codeword is not on the list (a decoding error), the condition will still not be satisfied for the transmitted codeword and a decoding error/failure is signaled. However if the condition is satisfied, then this implies that the transmitted codeword is on the ASD's list and, thus, a decoding success.

\section{B. Bounds on the ML Error Probability}

As important as it is to compare our algorithms with other algorithms, it is even more important to compare it with the ultimate performance limits, which is that of the soft-decision ML decoder. When transmitting the binary image of RS codes over a channel, the performance of the ML decoder depends on the weight enumerator of the transmitted binary image. The binary image of RS codes is not unique, but depends on the basis used to represent the symbols as bits. An average binary weight enumerator of RS codes could be derived by assuming a binomial distribution of the bits in a nonzero symbol [33]. Based on the Poltyrev tangential sphere bound (TSB) [34] and the average binary weight enumerator, average bounds on the ML error probability of RS codes over additive white Gaussian noise (AWGN) channels were developed in [33] and were shown to be tight. We will refer to this bound by ML-TSB. Alternatively the averaged binary weight enumerator could be used in conjunction with other tight bounds such as the Divsalar simple bound [35] to bound the ML error probability.

\section{Numerical Results}

In this section, we give some simulation results for our algorithm. As noted before, the multiplicity assignment algorithm used for ABP-ASD in the these simulations is the KV algorithm. $N 2$ denotes the number of outer iterations (parallel decoders) and $N 1$ is the number of inner iterations in each of these outer iterations.

1) $(15,11)$ RS Code Over an AWGN Channel: A standard binary input AWGN channel is assumed where the transmitted codewords are BPSK modulated. In Fig. 1, we compare the performance of different decoding algorithms. HD-BM refers to the performance of a hard decision bounded minimum distance decoder such as the BM algorithm. The ABP-BM list decoding algorithm with $N 1=5$ iterations and one iteration of LBP on each parity-check matrix, $I t_{H}=1$ (see step 4 in Alg. 2) has a coding gain of about $2.5 \mathrm{~dB}$ over HD-BM at a codeword error rate (CER) of $10^{-6}$. Increasing the number of iterations to $N 1=20$ iterations, we get a slightly better performance. $\mathrm{JN}-\mathrm{BM}$ refers to the $\mathrm{JN}$ algorithm with the JN stopping criterion and a $\mathrm{BM}$ decoder. Due to the high decoder error probability of the $(15,11)$ code, ABP-BM, with the list decoder stopping criterion, yields a much better performance than JN-BM. The ABP-ASD list decoding algorithm outperforms all the previous algorithms with only $5 \mathrm{ABP}$ iterations and with $I t_{H}=3$. Comparing its performance with soft-decision ML decoding of the RS code, we see that ABP-ASD has a near ML performance with a performance gain of about $3 \mathrm{~dB}$ over HD-BM at a CER of $10^{-6}$. (ML decoding was carried out by running the BCJR algorithm on the trellis associated with the binary parity-check 


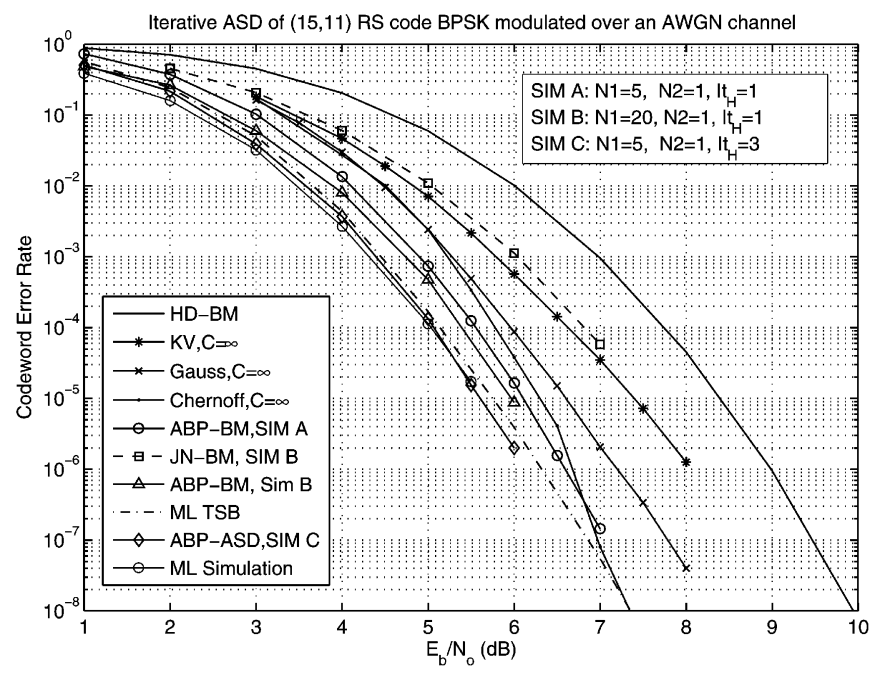

Fig. 1. Performance of iterative ASD of $(15,11)$ RS code, which is BPSK modulated and transmitted over an AWGN channel, is compared with that of other ASD algorithms and ABP-BM list decoding.

matrix of the RS code [36].) Moreover, the averaged TSB on the ML codeword error probability is shown to confirm that it is a tight upper bound and that the ABP-ASD algorithm is near-optimal for this code.

The performance of different ASD algorithms are compared for infinite interpolation costs, the KV algorithm [9], the Gaussian approximation (Gauss) [10], and the Chernoff bound algorithm (Chernoff) [11]. It is noted that the Chernoff bound algorithm has the best performance, especially at the tail of error probability. It is also interesting to compare the performance of ABP-ASD with other ASD MA algorithms. It has about $2 \mathrm{~dB}$ coding gain over the $\mathrm{KV}$ algorithm at a CER of $10^{-6}$. As expected, the Chernoff method has a comparable performance at the tail of the error probability.

The ABP algorithm used in the simulations shown in Fig. 1 is Alg. 2. The performance of Alg. 3 was identical to that of Alg. 2. However, the complexity is much less. The average $\delta$ [see (10)] averaged over all iterations was calculated versus the signal-to-noise ratio (SNR). It was observed that the ratio of the number of columns to be reduced in Alg. 3 to that in Alg. 2 is about $0.1(\delta=0.9)$. This gives about a $99 \%$ reduction in the Gaussian elimination complexity. Thus, only the first iteration or restart suffers from an Gaussian elimination complexity if Alg. 3 is used.

Near ML decoding for the same code is also achieved by the ABP-ASD algorithm with a finite cost of $10^{3}$, as shown in Fig. 2. Comparisons are made between the possible coding gains if the number of iterations is limited to $N 1=1,2,5$. With five iterations, the performance gain over the $\mathrm{KV}$ algorithm, with the same interpolation cost, is nearly $1.8 \mathrm{~dB}$ at a CER of $10^{-5}$. Comparing the ABP-ASD performance to that of Fig. 1, with infinite interpolation costs, we observe that a small loss in performance results with reasonable finite interpolation costs. Unless otherwise stated, the remaining simulations in this paper will assume infinite interpolation costs to show the potential of our algorithm.

It is to be noted that in simulating the ABP-BM list decoder, the simulations using a real BM decoder were identical to the

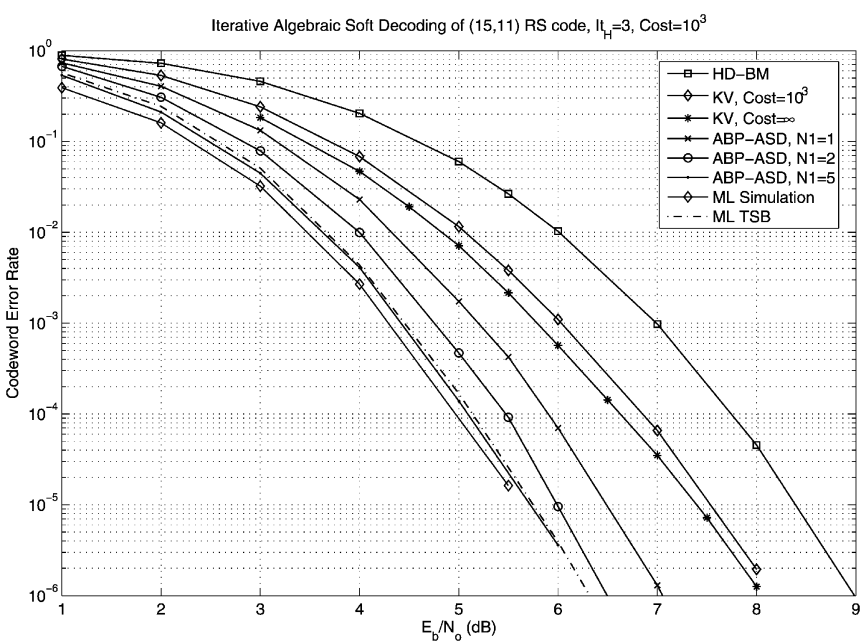

Fig. 2. Performance of iterative ASD of $(15,11)$ RS code, which is BPSK modulated and transmitted over an AWGN channel, is shown for a finite interpolation cost of $10^{3}$ and different iteration numbers.

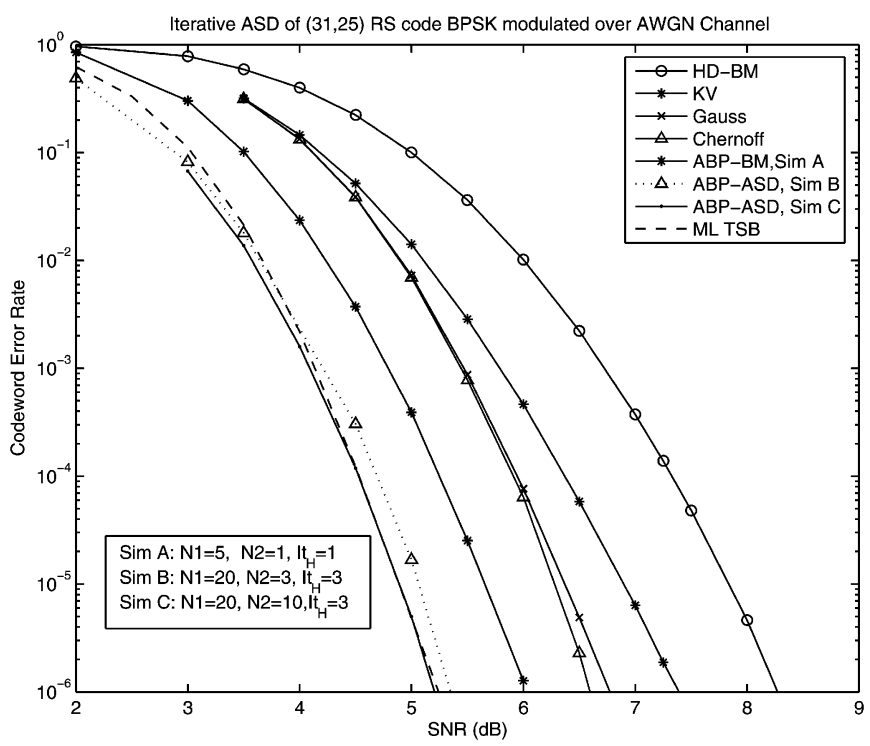

Fig. 3. ABP-ASD list decoding of the $(31,25)$ RS code transmitted over an AWGN with BPSK modulation.

simulations using the fast simulation setup described in this section. To save simulation time, the curves shown here for ABP-ASD are generated using the fast simulation setup. As is the case for ABP-BM, running the real ABP-ASD decoder will yield the same results.

2) $(31,25)$ RS Code Over AWGN Channel: The arguments for the $(15,11)$ RS code also carry over for the $(31,25)$ RS code when BPSK modulated and transmitted over an AWGN channel, as shown in Fig. 3. With only five iterations, the ABP-BM list decoding algorithm outperforms previous ASD algorithms. The performance of ABP-ASD with 20 inner iterations (N1) and 10 outer iterations (N2) is better than the ML upper bound and has more than $3 \mathrm{~dB}$ coding gain over the $\mathrm{BM}$ algorithm at an CER of $10^{-4}$. A favorable performance is also obtained by only three restarts (outer iterations). By comparing with Fig. 2 of [18], our ABP-ASD algorithm has about $1.6 \mathrm{~dB}$ gain over the combined Chase II-GMD algorithm at an CER of $10^{-4}$. 


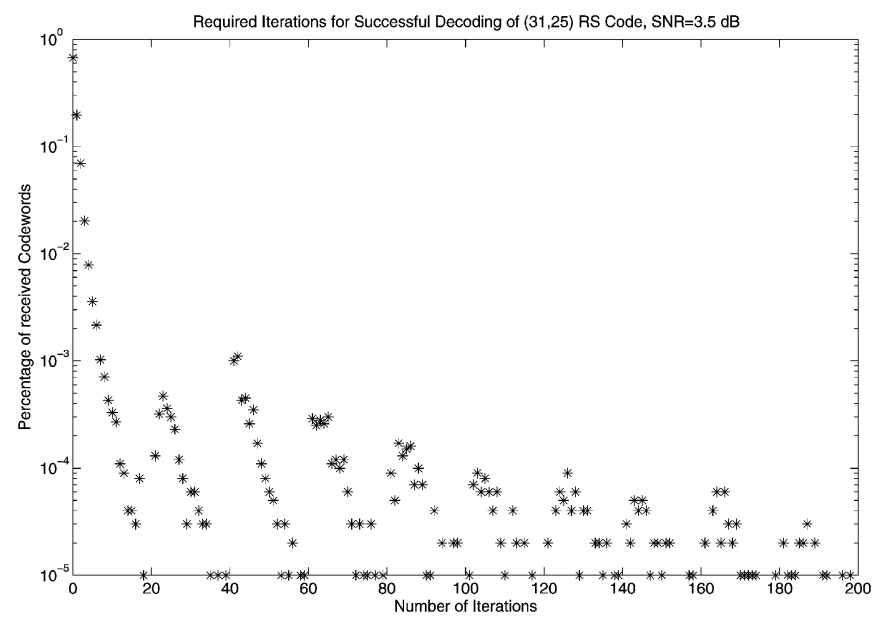

Fig. 4. Histogram shows the percentage of transmitted codewords successfully decoded versus the iteration number at which the transmitted codeword was first successfully added to the ABP-ASD list with $N 1=20$ and $N 2=10$. The $(31,25) \mathrm{RS}$ code is transmitted over an AWGN channel at an SNR of $3.5 \mathrm{~dB}$.

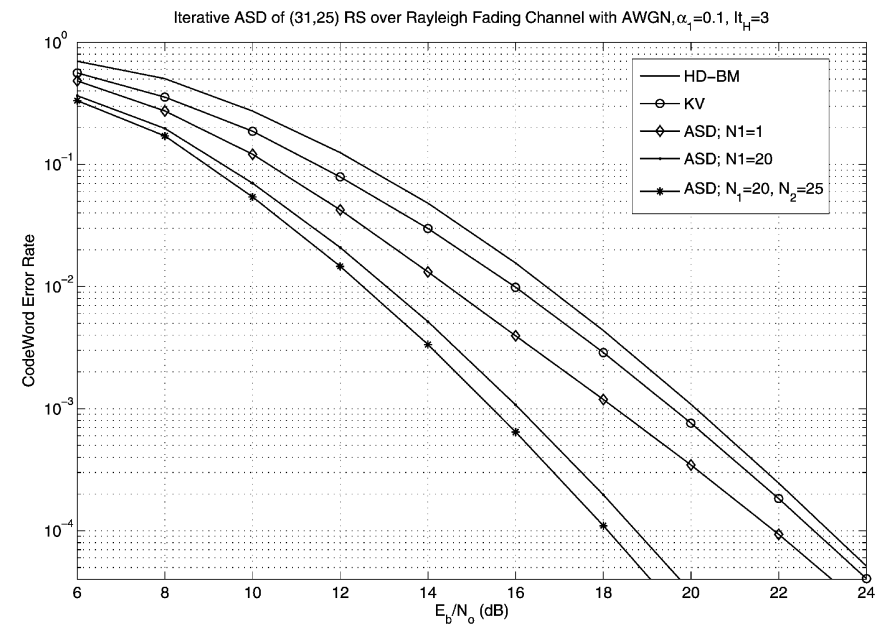

Fig. 5. Performance of the ABP-ASD decoding of the $(31,25)$ RS code over a Rayleigh-fading channel with AWGN when the channel information is unknown at the decoder.

To show the effectiveness of the restarts or outer iterations, we kept track of the iteration number at which the ABP-ASD list decoder was first capable to successfully decode the received word. In other words, this is the iteration when the transmitted codeword was first added to the ABP-ASD list. The percentage of transmitted codewords which were first successfully decoded at a certain iteration is plotted versus the iteration number in the histogram of Fig. 4. This is shown at an SNR of $3.5 \mathrm{~dB}$ and for $N 1=20 N 2=10$ with a total of 200 iterations. At the beginning of each restart (every 20 iterations), there is a boost in the number of codewords successfully decoded and this number declines again with increasing iterations. The zeroth iteration corresponds to the $\mathrm{KV}$ algorithm. This histogram is also invaluable for decoder design and could aid one to determine the designed number of iterations for a required CER.

3) $(31,25)$ RS Code Over a Rayleigh Fading Channel: As expected from the discussion in Section VI, the coding gain of ABP-ASD is much more if the underlying channel model is not memoryless. This is demonstrated in Fig. 5, where an $(31,25)$

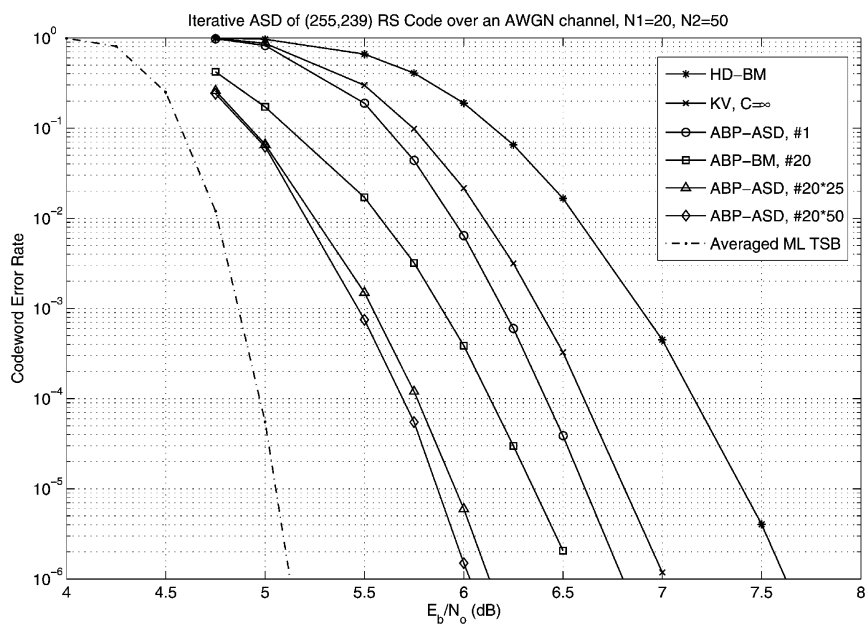

Fig. 6. Performance of the ABP-ASD decoding of the $(255,239)$ RS code over an AWGN channel with BPSK modulation.

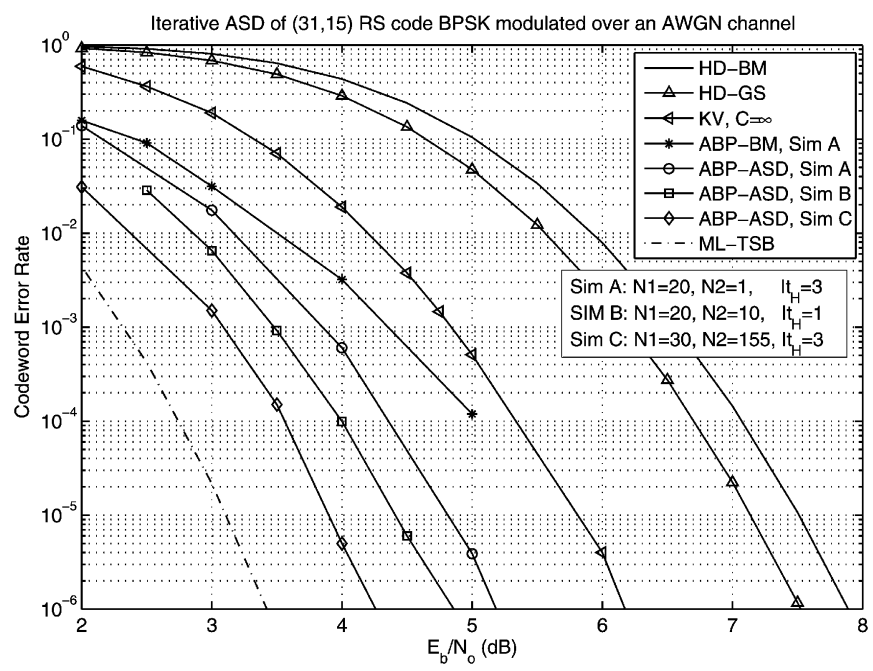

Fig. 7. ABP-ASD list decoding of the $(31,15)$ RS code, of rate 0.48 , transmitted over an AWGN with BPSK modulation.

code is BPSK modulated over a relatively fast Rayleigh-fading channel with AWGN. The Doppler frequency is equal to $50 \mathrm{~Hz}$ and the codeword duration is $0.02 \mathrm{~s}$. The coding gain of ABP-ASD over the KV algorithm at an CER of $10^{-4}$ is nearly $5 \mathrm{~dB}$ when the channel is unknown to both decoders.

4) $(255,239) R S$ Code Over AWGN Channel: The performance of the ABP-ASD algorithm is also investigated for relatively long codes. The $(255,239)$ code and its shortened version, the $(204,188)$ code, are standards in many communication systems. The performance of the $(255,239)$ code over an AWGN channel is shown in Fig. 6. By 20 iterations of ABP-BM, one could achieve a coding gain of about $0.5 \mathrm{~dB}$ over the $\mathrm{KV}$ algorithm. At an CER of $10^{-6}$, after a total of 25 outer iterations (restarts), the coding gain of ABP-ASD over BM is about $1.5 \mathrm{~dB}$. An extra $0.1 \mathrm{~dB}$ of coding gain is obtained with 25 more outer iterations. Moreover, the performance of the ABP-ASD decoder is within $1 \mathrm{~dB}$ of the averaged ML TSB.

5) $(31,15) R S$ Code Over AWGN Channel: The performance of our algorithm is studied for the $(31,15) \mathrm{RS}$ code over an AWGN channel. The rate of this code is 0.48 . Because this 
code is of relatively low rate, the HD-GS algorithm does improve over the HD-BM bounded minimum distance decoding algorithm. As seen from Fig. 7, ML soft-decision decoding offers about $4 \mathrm{~dB}$ coding gain over the hard decision GS algorithm and about $2.8 \mathrm{~dB}$ coding gain over the soft-decision KV ASD algorithm at an CER of $10^{-5}$. With 20 iterations, ABP-BM list decoding improves over the KV algorithm. As expected, ABP-ASD has a better performance for the same number of iterations. With 10 restarts, ABP-ASD has a reasonable performance with about a $3 \mathrm{~dB}$ coding gain over the $\mathrm{BM}$ algorithm. Another $0.5 \mathrm{~dB}$ of coding gain could be achieved by increasing the number of iterations.

6) General Observations: It is noticed that the coding gain between iterations decreases with the number of iterations. It is also to be noted that the ABP-ASD list decoder requires running the KV ASD algorithm in each iteration. Running a number of "plain-vanilla" ABP iterations without the ASD decoder and then decoding using the ASD decoder (to reduce the complexity) will yield a worse performance for the same number of iterations. The same arguments also hold for the ABP-BM list decoding. A reasonable performance is achieved by ABP-BM list decoding. By deploying the KV ASD algorithm, ABP-ASD list decoding has significant coding gains over the KV ASD algorithm and other well known soft-decision decoding algorithms.

\section{CONCLUSION}

In this paper, we proposed a list decoding algorithm for soft-decision decoding of RS codes. Our algorithm is based on enhancing the soft reliability channel information before passing them to an algebraic soft-decision decoding algorithm. This was achieved by deploying the Jiang and Narayanan algorithm, which runs belief-propagation on an adapted parity-check matrix. Using the Koetter-Vardy algorithm as the algebraic soft-decision decoding algorithm, our algorithm has impressive coding gains over previously known soft-decision decoding algorithms for RS codes. By comparing with averaged bounds on the performance of ML decoding of RS codes, we observe that our algorithm achieves a near optimal performance for relatively short, high-rate codes. We introduced some modifications over the $\mathrm{JN}$ algorithm that resulted in better coding gains. We presented a low complexity adaptive belief-propagation algorithm, which results in a significant reduction in the computational complexity. The performance of our algorithm was studied for the cases when the interpolation cost of the algebraic soft-decision decoding algorithm is both finite and infinite. A small loss in coding gain results when using manageable interpolation costs. The coding gain of the presented algorithm is larger for channels with memory. Our proposed algorithm could also be viewed as an interpolation multiplicity assignment algorithm for algebraic-soft decoding.

The question remains whether the JN algorithm is the optimum way to process the channel reliabilities before algebraic soft-decision decoding. The KV algorithm was our ASD decoder of choice due to its low complexity. Further investigations would be required to determine the best ASD algorithm or, in general, soft-decision decoding algorithm for joint belief-propagation list-decoding with an eye on both the performance and computational complexity.

\section{ACKNOWLEDGMENT}

The authors would like to thank J. Jiang and K. Narayanan for providing an extended version of their paper [13]. M. El-Khamy is grateful to M. Kan for confirming many of the simulation results in this paper. They gratefully acknowledge the comments of the anonymous reviewers that have improved the presentation of this paper.

\section{REFERENCES}

[1] I. S. Reed and G. Solomon, "Polynomial codes over certain finite fields," J. Soc. Ind. Appl. Math, vol. 8, pp. 300-304, 1960.

[2] P. Agashe, R. Rezaiifar, and P. Bender, "Cdma2000 high rate broadcast packet data air interface design," IEEE Commun. Mag., vol. 42, no. 2, pp. 83-89, Feb. 2004.

[3] E. R. Berlekamp, R. McEliece, and H. van Tilborg, "On the inherent intractability of certain coding problems," IEEE Trans. Inf. Theory, vol. 24, no. 3, pp. 384-386, May 1978.

[4] V. Guruswami and A. Vardy, "Maximum likelihood decoding of Reed Solomon codes is NP-hard," IEEE Trans. Inf. Theory, submitted for publication.

[5] A. Vardy and Y. Be'ery, "Bit level soft-decision decoding of ReedSolomon codes," IEEE Trans. Commun., vol. 39, no. 3, pp. 440-444, Mar. 1991

[6] V. Ponnampalam and B. Vucetic, "Soft decision decoding of Reed-Solomon codes," IEEE Trans. Commun., vol. 50, no. 22, pp. 1758-1768, Nov. 2002.

[7] V. Guruswami and M. Sudan, "Improved decoding of Reed-Solomon codes and algebraic geometry codes," IEEE Trans. Inf. Theory, vol. 45 pp. 1757-1767, Sep. 1999.

[8] M. Sudan, "Decoding of Reed-Solomon codes beyond the error-corrrection bound," J. Complexity, vol. 13, pp. 180-193, 1997.

[9] R. Kötter and A. Vardy, "Algebraic soft-decision decoding of ReedSolomon codes," IEEE Trans. Inf. Theory, vol. 49, no. 11, pp. 2809-2825, Nov. 2003.

[10] F. Parvaresh and A. Vardy, "Multiplicity assignments for algebraic softdecoding of Reed-Solomon codes," in Proc. Int. Symp. Inf. Theory, Jul. 2003 , p. 205

[11] M. El-Khamy and R. J. McEliece, "Interpolation multiplicity assignment algorithms for algebraic soft-decision decoding of Reed-Solomon codes," AMS-DIMACS, vol. 68, pp. 99-120, 2005. Vol. on Algebraic Coding Theory and Information Theory.

[12] M. El-Khamy, R. McEliece, and J. Harel, "Performance enhancements for algebraic soft-decision decoding of Reed-Solomon codes," in Proc. Int. Symp. Inf. Theory, 2004, p. 421.

[13] J. Jiang and K. Narayanan, "Iterative soft decision decoding of Reed Solomon codes based on adaptive parity-check matrices," in Proc. Int Symp. Inf. Theory, Jul. 2004, p. 261.

[14] — "Iterative soft decision decoding of Reed-Solomon codes," IEEE Commun. Lett., vol. 8, pp. 244-246, Apr. 2004.

[15] M. Fossorier and S. Lin, "Soft-decision decoding of linear block codes based on ordered statistics," IEEE Trans. Inf. Theory, vol. 41, no. 5, pp. 1379-1396, Sep. 1995.

[16] G. D. Forney, "Generalized minimum distance decoding," IEEE Trans. Inf. Theory, vol. IT-12, no. 2, pp. 125-131, Apr. 1966.

[17] D. Chase, "A class of algorithms for decoding block codes with channel measurement information," IEEE Trans. Inf. Theory, vol. IT-18, no. 1, pp. 170-182, Jan. 1972.

[18] H. Tang, Y. Liu, M. Fossorier, and S. Lin, "On combining chase-2 and GMD decoding algorithms for nonbinary block codes," IEEE Commun. Lett., vol. 5, no. 5, pp. 209-211, May 2001.

[19] T.-H. Hu and S. Lin, "An efficient hybrid decoding algorithm for ReedSolomon codes based on bit reliability," IEEE Trans. Commun., vol. 51, no. 7, pp. 1073-1081, Jul. 2003.

[20] F. J. MacWilliams and N. J. Sloane, The Theory of Error Correcting Codes. Amsterdam, The Netherlands: North Holland, 1977.

[21] R. J. McEliece, The Theory of Information and Coding, 2nd ed. Cambridge, U.K.: Cambridge Univ. Press, 2002.

[22] R. Horn and C. Johnson, Matrix Analysis. Cambridge, U.K.: Cambridge Univ. Press, 1985. 
[23] R. J. McEliece, "The Guruswami-Sudan decoding algorithm for ReedSolomon codes," IPN Progress Rep., Tech. Rep. 42-153, 2003.

[24] R. Nielsen and T. Hoeholdt, "Decoding Reed-Solomon codes beyond half the minimum distance," in Cryptography and Related Areas, J. Buchmann, T. Hoeholdt, H. Stichenoth, and H. Tapia-Recillas, Eds. New York: Springer-Verlag, 2000, pp. 221-236.

[25] W. J. Gross, F. R. Kschischang, R. Kötter, and P. G. GulakR, "Applications of algebraic soft-decision decoding of Reed-Solomon codes," IEEE Trans. Commun., Jul. 2003, submitted for publication.

[26] R. Gallager, Low Density Parity Check Codes. Cambridge, MA: MIT Press, 1963.

[27] R. McEliece, D. MacKay, and J. Cheng, "Turbo decoding as an instance of Pearl's belief propagation algorithm," IEEE J. Sel. Areas. Commun., vol. 16, pp. 140-152, Feb. 1998.

[28] J. Hagenauer, E. Offer, and L. Papke, "Iterative decoding of binary block and convolutional codes," IEEE Trans. Inf. Theory, vol. 42, pp. 429-449, Mar. 1996.

[29] J. Yedidia, W. Freeman, and Y. Weiss, "Understanding belief propagation and its generalizations," in Exploring Artif. Intell. New Millennium, 2003, ch. 8, pp. 239-236.

[30] A. Ahmed, R. Koetter, and N. R. Shanbhag, "Performance analysis of the adaptive parity check matrix based soft-decision decoding algorithm," in Proc. 38th Asilomar Conf. Signals, Syst., Comput., vol. 2, 2004, pp. 1995-1999.

[31] R. Roth and G. Ruckenstein, "Efficient decoding of Reed-Solomon codes beyond half the minimum distance," IEEE Trans. Inf. Theory, vol. 46, no. 1, pp. 246-257, Jan. 2000.

[32] R. J. McEliece, "On the average list size for the Guruswami-Sudan decoder," in Proc. 7th Int. Symp. Commun. Theory Appl., 2005, pp. 2-6.

[33] M. El-Khamy and R. J. McEliece, "Bounds on the average binary minimum distance and the maximum likelihood performance of Reed Solomon codes," in Proc. 42nd Allerton Conf. Commun., Control Comput., 2004, pp. 290-299.

[34] G. Poltyrev, "Bounds on the decoding error probability of binary linear codes via their spectra," IEEE Trans. Inf. Theory, vol. 40, no. 4, pp. 1284-1292, Jul. 1994.

[35] D. Divsalar, "A simple tight bound on error probability of block codes with application to turbo codes," TMO Progress Rep., NASA, JPL, Tech. Rep. 42-139, 1999.

[36] M. Kan, Private Communication.

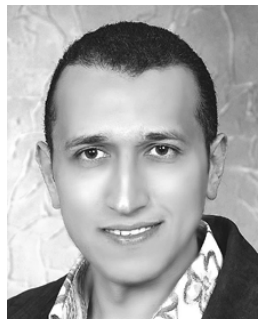

Mostafa El-Khamy (S'02) received the B.S. and M.S. degrees from the University of Alexandria, Alexandria, Egypt, in 1999 and 2001, respectively, and the M.S. degree from the California Institute of Technology, Pasadena, in 2003, all in electrical engineering. He is currently working towards the Ph.D. degree in the Caltech Communications Group, California Institute of Technology.

During the summer of 2005, he worked as an Engineering Intern at Corporate Research and Development, Qualcomm, San Diego, CA. His research interests include information theory, coding theory, multiuser detection, and wireless communication systems.

Mr. El-Khamy received the Atwood Fellowship from the California Institute of Technology in 2002. He was also the recipient of the Young Scientist Award from the General Assembly of the International Union of Radio Science (URSI), Maastricht, The Netherlands, in 2002.

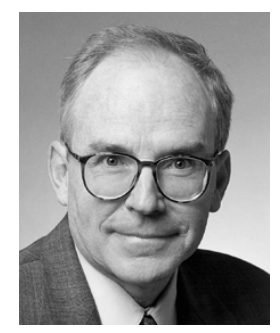

Robert J. McEliece (M'70-SM'81-F'84) has been on the faculty at the California Institute of Technology, Pasadena, since 1982, where he is now the Allen E. Puckett Professor and Professor of Electrical Engineering. From 1990 to 1999, he served as Executive Officer for Electrical Engineering at the California Institute of Technology. He has been a Consultant in the Communications Research Section of the Jet Propulsion Laboratory since 1978. His research interests include deep-space communication, communication networks, coding theory, and discrete mathematics.

Dr. McEliece is a member of the National Academy of Engineering. He was the recipient of the 2004 IEEE Information Theory Society Shannon Award. 